\section{ORAL PATHOLOGY}

\section{Osteonecrosis of the jaws in patients} treated with biphosphonates histomorphologic analysis in comparison with infected osteoradionecrosis

Hansen T, Kunkel M et al. J Oral Pathol Med 2006; 35: 155-160

This study suggests that Actinomyces is significantly involved in both diseases.

Osseous metastases may cause severe pain and pathological fractures, and biphosphonates are used to inhibit osteolysis. A complication of this treatment is osteonecrosis occurring almost exclusively in the jaw, and the present study compared 8 patients with this condition and 10 with jaw osteoradionecrosis following radiotherapy for tumours. In all cases, biopsies were taken.

In most cases in both groups, inflammatory infiltrates were found, along with medullary space fibrosis. There was minimal obliteration of blood vessels, and in all cases in both groups, Actinomyces was found, commonly at sites of eroded necrotic bone. In one case, fungal spores were also present. There were also some possible differences between the groups. The authors discuss their findings, and consider that Actinomyces may be involved in the chronic, non-healing processes which are present.

doi:10.1038/sj.bdj.4813675

ENDODONTICS

Incidence of periapical lesions and clinical symptoms after pulpectomy - a clinical and radiographic evaluation of 1 - versus 2session treatment

Gesi A, Hakeberg M etal. Oral Surg 2006; 101:379-388

Given meticulous treatment, there appeared to be no difference in outcome of the two groups.

One visit treatment of vital pulp conditions may be appropriate because infection is more limited than with a non-vital condition. This randomised trial compared 1- and 2-visit treatment of vital pulp conditions. From 295 patients assessed, 256 were accepted and randomised to the groups, and 12 were wholly lost to follow-up; 90\% attended at 2- or 3-yr recalls or both; 72\% attended the 3-yr recall. The 2-visit treatment used a calcium hydroxide dressing between visits.

Pain was reported in 80\% of patients preoperatively, and in 13\% postoperatively. Most postoperative pain was in teeth which had been overfilled $(P<0.001)$, and pain did not differ significantly between groups. Preoperative radiography identified small periapical radiolucencies in $16 \%$ of patients, evenly divided between groups. Periapical lesions were present in 7\% (17) of patients at 3 yrs and were evenly distributed between groups. In 13 patients, the lesions had been observed at $1 \mathrm{yr}$. The authors conclude that with aseptic conditions and proper instrumentation and filling, the 2-visit approach had no advantage.

\section{ORAL PATHOLOGY}

\section{Central giant cell granuloma of the jaws: a clinical, radiologic and histopathologic study of 26 cases}

Kruse-Lösler B, Diallo R et al. Oral Surg 2006; 101: 346-354

This study adds further information towards predicting aggressive behaviour of central giant cell granuloma (CGCG).

CGCG is a rare lesion, behaving in a way which is often unpredictable, varying from a slowly swelling to a highly aggressive, painful lesion. Opinions differ on whether it is neoplastic or reactive. This study was a retrospective examination of 26 patients with CGCG seen in a German hospital over a 24 year period. The lesions were classified as nonaggressive (NA; mean age and range: 8.6 yrs, 4.8-14.0) or aggressive (A; 15.3, 6.0-57.4), and their characteristics compared.

Females accounted for $2 / 3$ of all cases, and 2/3 also were in the mandible. Group A lesions appeared much larger at diagnosis (mean diameter 56 v. $33 \mathrm{~mm}$ ). In group A, pain, rapid growth, root resorption and displacement were common; in group NA, these features did not occur. There were no recurrences in group NA, but 4 in group A. Surgical removal was undertaken in all cases: curettage in 5 cases, curettage with ostectomy in 20, and extra-oral block resection in one. The authors consider that the clinical and histological features may help predict the behaviour of the lesions.

doi:10.1038/sj.bdj.4813677

\section{ORAL MEDICINE}

On the natural course of oral lichen lesions in a Swedish population-based sample

Roosaar A, Yin L et al. J Oral Pathol Med 2006; 35: 257-261

The natural course appeared benign in the great majority of lesions in this cohort.

Oral lichen planus (OLP) is classed as a precancerous condition, but there is conflicting evidence on this matter from different studies. In 1973-4, subjects were investigated for oral mucosal lesions in a Swedish county, and a cohort of 327 with oral lichen lesions (OLLs) was identified. (In 1973-4, no distinction was made between OLP and oral lichenoid reaction, so the two categories were combined as OLL in this study.)

In 1993-4, a sample of 80 subjects still living in the area was selected from the remaining 194, and 55 of them were re-examined. In this sample, 14 of 36 initial white lesions had resolved, while 4 had turned to red lesions. In 19 red lesions, 5 had resolved, and 4 had turned white. No malignancies were observed.

For the whole cohort, in 2002, incidence of specified cancers (SIRs) and mortality from all and specific causes (SMRs) was identified from the excellent Swedish registries, and none was significantly elevated. The authors consider that OLLs carry a low risk of harm.

doi:10.1038/sj.bdj.4813678 